Bundesgesundheitsbl $2021 \cdot 64: 838-846$ https://doi.org/10.1007/s00103-021-03349-2 Eingegangen: 1. Februar 2021

Angenommen: 6. Mai 2021

Online publiziert: 20. Mai 2021

(c) Der/die Autor(en) 2021

\section{Anja Heilmann' · Sebastian Ziller ${ }^{2}$}

' Department of Epidemiology and Public Health, University College London, London, Großbritannien

${ }^{2}$ Abteilung Prävention und Gesundheitsförderung, Bundeszahnärztekammer (BZÄK), Berlin, Deutschland

\title{
Reduzierung des Zuckerkonsums für eine bessere Mundgesundheit - Welche Strategien sind Erfolg versprechend?
}

\section{Einleitung}

Im Januar 2021 verabschiedete der Exekutivrat (EB148) der Weltgesundheitsorganisation (WHO) mit breiter Unterstützung eine Resolution zur Mundgesundheit - laut WHO-Generalsekretär Dr. Tedros in der Geschichte der Mundgesundheit ein wegweisender Meilenstein. Nachdem die Mundgesundheit über Jahrzehnte hinweg nur eine geringe gesundheitspolitische Priorität genoss, spiegelt die aktuelle Resolution ein Umdenken auf höchster Ebene wider, das die Mundgesundheit als einen wesentlichen und untrennbaren Bestandteil der Allgemeingesundheit anerkennt [1]. Die Zahlen sprechen für sich: Unbehandelte Karies der bleibenden Zähne ist die weltweit häufigste aller chronischen, nichtübertragbaren Erkrankungen. Im Jahr 2015 wurde die Zahl der weltweit Betroffenen auf 2,5 Mrd. und die altersstandardisierte Prävalenz auf etwa $34 \%$ geschätzt [2]. Zudem gibt es bei der Kariesverteilung in der Bevölkerung in allen Altersgruppen ein starkes soziales Gefälle [3]. Auch in Deutschland ist Karies weitverbreitet. Während laut Fünfter Deutscher Mundgesundheitsstudie (DMS V) die Kariesprävalenz bei Kindern und Jugendlichen über die vergangenen Jahrzehnte erfreulicherweise stark zurückgegangen ist und $81 \%$ der 12-Jährigen 2015 kariesfrei waren, wurde bei knapp $25 \%$ der jungen Erwachsenen sanierungsbedürftige Karies festgestellt und weniger als 3\% hatten keine Karieserfahrung [4].

Karies ist keine harmlose Erkrankung - sie beeinträchtigt Gebissfunktion und Ästhetik und kann die Lebensqualität erheblich mindern. Karies verursacht Schmerzen und kann vor allem bei Kindern zu Appetitverlust, Schlaflosigkeit, Konzentrationsstörungen sowie Schulabwesenheit und sogar Gewichtsverlust führen [5]. Karies ist neben der Parodontitis eine der Hauptursachen für Zahnverlust und ihre Behandlung ist zeitaufwendig und teuer.

Karies ist jedoch überwiegend vermeidbar. Die entscheidende Ursache für die Entstehung von Karies ist übermäßiger Zuckerkonsum $[6,7]$. Es ist wichtig, diese Tatsache klar hervorzuheben. Faktoren wie Speichelfluss und Aufnahme von Fluoriden wirken dem Kariesprozess entgegen und spielen somit eine wichtige Rolle, werden aber als Effektmodifikatoren angesehen [6]. Der Zuckerkonsum selbst wird wiederum durch umweltbedingte und psychosoziale Faktoren bestimmt [8].

Ein hoher Zuckerkonsum ist auch mit einem erhöhten Risiko für Parodontalerkrankungen assoziiert. Es wird angenommen, dass durch die Nahrung aufgenommener Zucker chronisch entzündliche Erkrankungen wie Parodontitis begünstigt [9].

Die wirtschaftlichen Kosten für die Behandlung von Karies, Parodontitis und Zahnverlust sind immens. Laut ei- ner Modellrechnung beliefen sich die direkten und indirekten Kosten von durch übermäßigen Zuckerkonsum verursachten Zahnerkrankungen im Jahr 2010 auf global 172 Mrd. US-Dollar. Für Deutschland wurden dabei pro Jahr und Person $210 €$ an Zahnbehandlungskosten errechnet [10]. Angesichts der erheblichen Beeinträchtigungen der Lebensqualität für den Einzelnen sowie der hohen Kosten für Gesundheitssystem und Gesellschaft stellen diese zuckerbedingten Erkrankungen also ein signifikantes Gesundheitsproblem dar.

Zucker spielt aber nicht nur für die Mundgesundheit eine Schlüsselrolle. Ein hoher Zuckerkonsum, vor allem durch zuckrige Getränke, geht mit einem erhöhten Risiko für die Entstehung von Übergewicht und Adipositas sowie deren Folgeerkrankungen einher [11]. Wissenschaftlich belegt sind außerdem Zusammenhänge zwischen Zuckerkonsum und Diabetes Typ 2 [12] sowie Herz-Kreislauf-Erkrankungen [13]. Als Risikofaktor lange vernachlässigt, ist Zucker infolge dieser Erkenntnisse mehr und mehr ins Blickfeld der Gesundheitspolitik gerückt.

In diesem Beitrag beschreiben wir die derzeit geltenden Empfehlungen der WHO zum Zuckerkonsum sowie Daten zum Verzehr in Deutschland. Unser Ziel ist es, wichtige Faktoren, die den Zuckerkonsum beeinflussen, zu beleuchten und Konzepte und Strategien zur Zuckerreduzierung darzulegen, um die Mundgesundheit zu verbessern. 


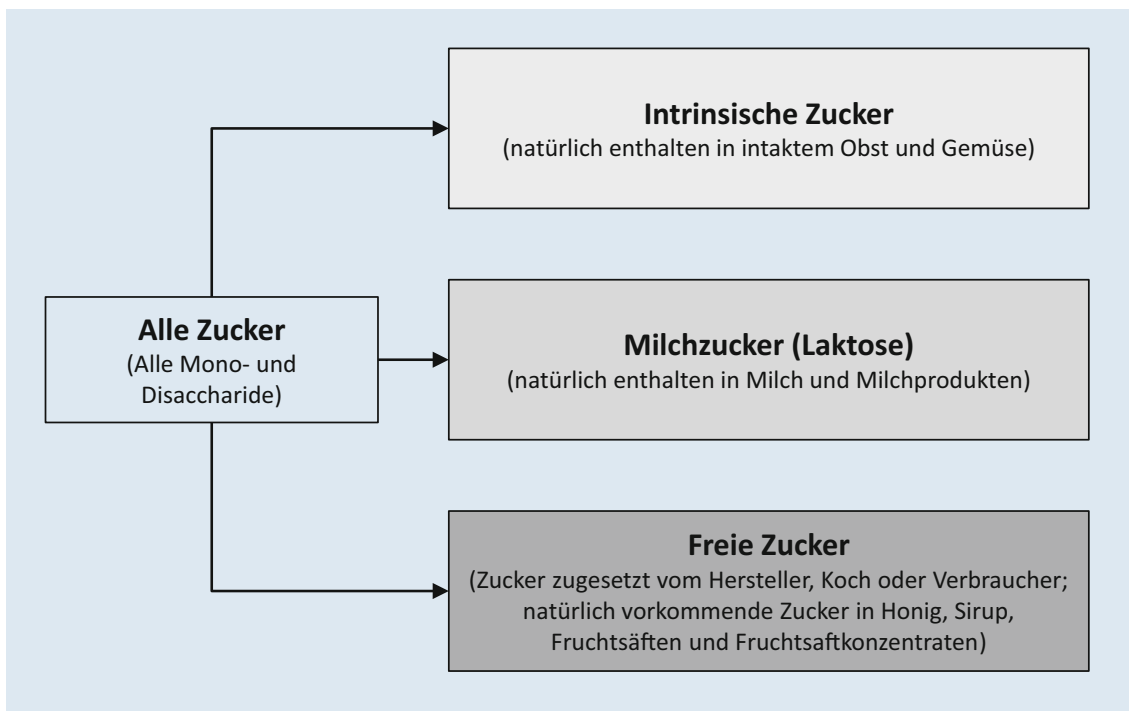

Abb. 1 ॥ Zuckerklassifikation (Abbildung übersetzt aus Heilmann et al. [8])

\section{WHO-Richtlinie zum Zucker- konsum}

Im Jahr 2015 wurde von der WHO eine evidenzbasierte Richtlinie zum Zuckerkonsum veröffentlicht [14]. Die WHO unterscheidet zwischen intrinsischem Zucker, der in frischem, intaktem Obst und Gemüse vorkommt, Milchzucker (Laktose), der natürlich in Milch und Milchproduckten enthalten ist, und dem sogenannten freien Zucker (• Abb. 1). Die WHO-Richtlinie bezieht sich ausschließlich auf freie Zucker. Die Definition freier Zucker umfasst alle Mono- und Disaccharide, die Lebensmitteln und Getränken vom Hersteller, Koch oder Verbraucher hinzugefügt werden, sowie die natürlich vorkommenden Zucker in Honig, Sirup, Fruchtsäften und Fruchtsaftkonzentraten [14]. Im Gegensatz zu den freien Zuckern gelten sowohl Milchzucker als auch die in frischem Obst und Gemüse ${ }^{1}$ enthaltenen Zucker als gesundheitlich unbedenklich [14].

Die WHO-Richtlinie basiert hauptsächlich auf 2 systematischen Reviews, welche den Stand der epidemiologischen Forschung zur Bedeutung freier Zucker sowohl für ungesunde Gewichtszunah-

\footnotetext{
1 "Frisch" bedeutet hier ganzes Obst und Gemüse mit intakter Zellstruktur, schließt also Fruchtsäfte oder getrocknetes Obst nicht mit ein.
}

me [11] als auch Zahnkaries [7] zusammenfassen.

Um das Risiko von Übergewicht und dessen Folgeerkrankungen sowie von Karies zu reduzieren, empfiehlt die WHO, die Aufnahme freier Zucker in allen Lebensphasen auf unter 10 Energieprozent zu senken (nachdrückliche Empfehlung). Bei einer Aufnahme von 2000 Kilokalorien pro Tag entspricht dies rund $50 \mathrm{~g}$ Zucker (ca. 10 Teelöffel) für einen durchschnittlichen Erwachsenen. Zusätzlich hält die WHO eine weitere Reduktion auf unter 5 Energieprozent (also täglich nicht mehr als 5 Teelöffel bzw. $25 \mathrm{~g}$ Zucker für Erwachsene) für sinnvoll (ergänzende Empfehlung; $[14])^{2}$

Die WHO-Empfehlungen stellen für nationale, gesundheitspolitische Entscheidungsträger einen Referenzrahmen dar und sollen eine treibende Kraft für gesundheitspolitische Veränderungen in Richtung Zuckerreduktion sein [15]. In Deutschland haben die Deutsche Adipositas-Gesellschaft e.V., die Deutsche Diabetes Gesellschaft e. V. und die Deut-

\footnotetext{
2 Nachdrückliche Empfehlungen werden von der WHO ausgesprochen, wenn die Forschungslage zeigt, dass bei Einhaltung der Empfehlung die erwünschten Effekte überwiegen und die Empfehlung in den meisten Situationen umsetzbar ist. Ergänzende Empfehlungen werden dann ausgesprochen, wenn über die Balance zwischen Vor- und Nachteilen der Umsetzung weniger Sicherheit besteht [14].
}

sche Gesellschaft für Ernährung e.V. ein Konsenspapier veröffentlicht, in dem sie sich der nachdrücklichen WHOEmpfehlung für eine maximale Zufuhr freier Zucker von weniger als $10 \%$ der Gesamtenergiezufuhr anschließen [16].

\section{Zuckerkonsum in Deutschland}

$\mathrm{Zu}$ den wichtigsten Quellen freier $\mathrm{Zu}$ cker in der Ernährung der Deutschen gehören zuckerhaltige Getränke, Süßigkeiten und Backwaren [17]. Für Kinder spielen Frühstückszerealien mit hohem Zuckergehalt eine wichtige Rolle [18].

Repräsentative Daten zum Zuckerkonsum der erwachsenen deutschen Bevölkerung wurden zuletzt durch die zwischen 2005 und 2007 durchgeführte Nationale Verzehrsstudie II erfasst. Demnach lag der durchschnittliche Verzehr freier Zucker für Männer bei täglich $78 \mathrm{~g}$ oder 13 Energieprozent und für Frauen bei $61 \mathrm{~g}$ oder 14 Energieprozent. Der Zuckerkonsum ist jedoch stark altersabhängig. Bei den Männern war er am höchsten bei den 15- bis 24-Jährigen mit durchschnittlich 17 Energieprozent pro Tag und am niedrigsten bei den 65 - bis 80-Jährigen mit etwa 10 Energieprozent pro Tag. Bei den Frauen lagen die entsprechenden Werte bei $18 \%$ und $12 \%$ [17]. Vor allem für junge Erwachsene sind diese Werte weitaus höher als das von der WHO empfohlene Maximum von 10 oder gar 5 Energieprozent.

Die Messung des Konsums freier $\mathrm{Zu}$ cker bei Kindern (3-18 Jahre) in der Studie DONALD (Dortmund Nutritional and Anthropometric Longitudinally Designed) ergab für den Zeitraum zwischen 2005 und 2016 einen Wert von durchschnittlich 16-17 Energieprozent für Mädchen und Jungen. Laut DONALD war der Verzehr bei jüngeren Kindern höher als bei älteren. Zwischen 2010 und 2016 verzeichnete die Studie einen erfreulichen Abwärtstrend, allerdings blieb der Zuckerkonsum nach wie vor deutlich über der von der WHO empfohlenen Menge [19].

Es gibt deutliche Hinweise, dass der Zuckerkonsum bei Kindern und Jugendlichen sozial ungleich verteilt ist. - Abb. 2 zeigt Daten der Studie zur Gesundheit von Kindern und Jugendli- 
chen in Deutschland (KiGGS) Welle 2 (2014-2017) für den täglichen Konsum zuckerhaltiger Erfrischungsgetränke (z. B. Cola, Limonade; Fruchtsäfte nicht eingeschlossen). Die Daten dokumentieren ein klares soziales Gefälle: Ein täglicher Konsum wurde weit häufiger für Kinder aus Familien mit niedrigem sozioökonomischen Status (SES) angegeben als für Gleichaltrige aus Familien mit höherem SES [20].

\section{Wodurch wird der Zucker- konsum beeinflusst?}

\section{Evolution und globaler Ernährungswandel}

Menschen sind durch die Evolution auf eine Vorliebe für süße, fetthaltige, energiereiche Nahrung programmiert für unsere steinzeitlichen Vorfahren brachten diese Präferenzen einen klaren Überlebensvorteil. Durch den globalen Ernährungswandel leben wir heute allerdings in einer Welt, in der zuckrige und hochkalorische Nahrungsmittel jederzeit billig verfügbar sind und auch stark beworben werden. Dieses Missverhältnis zwischen inhärenten Vorlieben und einer modernen Umwelt, in der diese Vorlieben für Krankheiten anfällig machen, ist ein wesentlicher Grund für die weltweit starke Zunahme chronischer Erkrankungen über die vergangenen Jahrzehnte [21]. Es erklärt auch den nur mäßigen Erfolg von Maßnahmen, die allein auf individuelle Verhaltensänderung durch Gesundheitserziehung abzielen, aber Lebens- und Konsumwelten unangetastet lassen [22, 23].

\section{Soziale Gesundheitsdeterminan-} ten

Wenn es um Gesundheitsverhalten geht, wird häufig von „Lebensstil“ (Lifestyle) gesprochen, als wären dies Entscheidungen, die unabhängig von den Lebensumständen in einem "Vakuum“ getroffen werden $[8,24]$. Dabei wird oft übersehen, dass nicht alle Menschen über die gleichen Ressourcen und Entscheidungsmöglichkeiten verfügen. Menschliches Verhalten wird von unzähligen Faktoren geprägt, von denen viele außerhalb der

Bundesgesundheitsbl 2021 · 64:838-846 https://doi.org/10.1007/s00103-021-03349-2

(c) Der/die Autor(en) 2021

\section{A. Heilmann · S. Ziller \\ Reduzierung des Zuckerkonsums für eine bessere Mundgesundheit - Welche Strategien sind Erfolg versprechend?}

\section{Zusammenfassung}

Strategien zur Gesundheitsförderung können einen wesentlichen Beitrag leisten, um nichtübertragbare chronische Krankheiten zu verhindern. Die wesentlichen nichtübertragbaren Erkrankungen in der Zahnmedizin sind Zahnkaries und Parodontalerkrankungen. Zucker spielt sowohl bei der Entstehung von Zahnkaries als auch von Übergewicht und dessen Folgen für die Allgemeingesundheit eine ursächliche Rolle und ist daher als wichtige kommerzielle Gesundheitsdeterminante mehr und mehr ins Blickfeld von Wissenschaft und Gesundheitspolitik gerückt. Existierende Strategien zur Reduktion des Zuckerkonsums zielen jedoch häufig auf Maßnahmen zur individuellen Verhaltensänderung ab und lassen dabei die Rolle von gesellschaftlichen und kommerziellen Einflüssen außer Acht. In diesem Artikel beschreiben wir die aktuellen Empfehlungen der Weltgesund- heitsorganisation (WHO) zum Zuckerkonsum, Daten zum Zuckerverzehr in Deutschland sowie die sozialen und kommerziellen Faktoren, welche den Zuckerkonsum beeinflussen. Grundlegende Prinzipien der Gesundheitsförderung werden dargelegt und sich daraus ergebende Strategien zur Zuckerreduzierung diskutiert. Dabei werden konkrete Beispiele für Upstreamund Downstream-Ansätze benannt und Möglichkeiten der Einflussnahme durch die zahnmedizinische Community in Politik und Praxis aufgezeigt.

\section{Schlüsselwörter}

Zucker · Mundgesundheit · Karies · Kommerzielle Gesundheitsdeterminanten . Gesundheitsstrategien

\section{Reducing sugar consumption to improve oral health-which strategies are effective?}

\section{Abstract}

Health promotion strategies can make a significant contribution to the prevention of non-communicable diseases. The most important non-communicable diseases affecting the mouth and teeth are dental caries and periodontal disease. Sugar is a causal factor in the development of dental caries, obesity and obesity-related diseases. Sugar is therefore recognised as an important commercial determinant of health and a public health priority. However, existing strategies to reduce sugar consumption often focus on individual behaviour change while ignoring the role of the wider social and commercial environment.

In this article we describe the current recommendations on sugar intake made by the World Health Organization, existing data on sugar intake in Germany as well as the social and commercial factors that influence sugar consumption. Important public health principles will be outlined, followed by a discussion of appropriate sugar reduction strategies. Examples for upstream and downstream approaches to prevention are presented and opportunities for dental health professionals to influence policy and practice highlighted.

\section{Keywords}

Sugar consumption - Oral health - Dental caries - Commercial determinants of health . Public health strategies
Kontrolle des Einzelnen liegen [25]. Diese Faktoren werden unter dem Begriff der sozialen Gesundheitsdeterminanten zusammengefasst und erklären die in fast allen Gesellschaften anzutreffenden sozialen Ungleichheiten bei der Verteilung von Krankheit und Gesundheit [26]. Auch der Zuckerkonsum wird von einem komplexen Zusammenspiel sich gegenseitig beeinflussender Gegebenheiten bestimmt [8]. Auf oberster Ebene gehören dazu z.B. die wirtschaftlichen Rahmenbedingungen, die für Produktion, Zusammensetzung, Handel, Verfügbarkeit und Erschwinglichkeit von zuckerhaltigen Produkten entscheidend sind. Ebenso wichtig sind die Marketingstrategien der Lebensmittelindustrie 


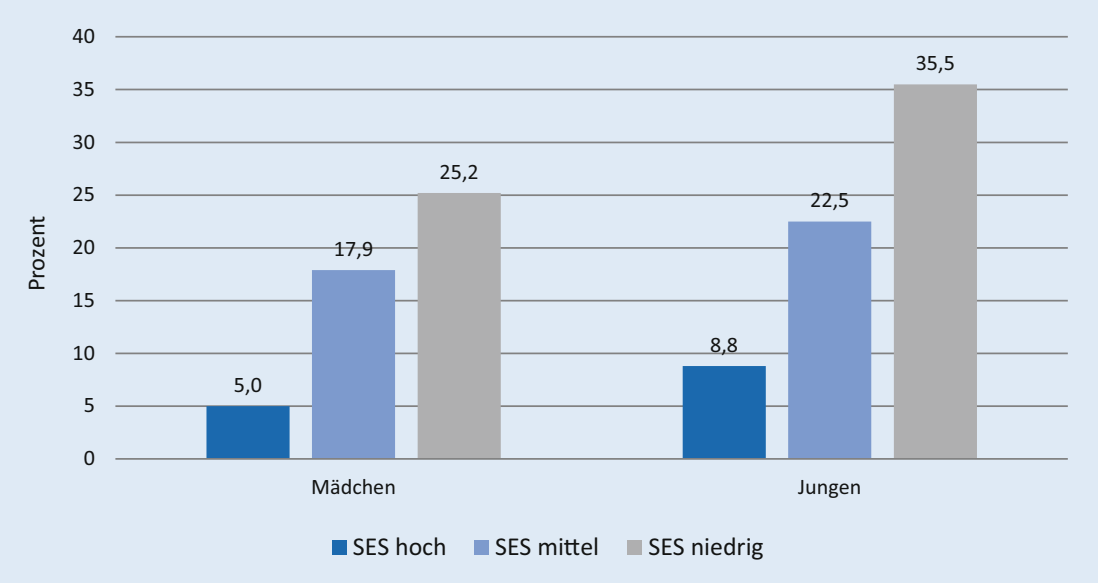

Abb. 2 \ Täglicher Konsum zuckerhaltiger Erfrischungsgetränke bei Kindern und Jugendlichen in Deutschland 2014-2017 (KiGGS Welle 2), aufgeschlüsselt nach Geschlecht und sozioökonomischem Status (SES). (Datenquelle: KiGGS Welle 2, aus [20])

sowie der Umfang, zu dem diese vom Staat reguliert werden [27]. Das Angebot von Speisen und Getränken in Lebenswelten wie Schulen und Arbeitsplätzen bestimmt maßgeblich, was dort konsumiert wird, und kann zu sozialen Normen beitragen. Auf individueller Ebene spielen Einkaufsmöglichkeiten im Wohngebiet und verfügbares Einkommen eine Rolle - so sind (bei gleicher Menge an Kalorien) frisches Obst und Gemüse vergleichsweise teurer als zuckrige und fettreiche Produkte [28]. Ebenfalls wichtig sind die Erwartungen von Familie und Freunden, aber auch ernährungsbezogenes Wissen sowie die Fähigkeit und Möglichkeit, gesundes Essen selbst zu kochen. Nicht $\mathrm{zu}$ vernachlässigen sind psychosoziale Faktoren - für Menschen, die unter viel Stress stehen, kann süßes Essen ein Bewältigungsmechanismus sein. Außerdem besteht ein Zusammenhang zwischen depressiver Symptomatik und Essverhalten [29].

\section{Zucker als kommerzielle Gesundheitsdeterminante}

Das Konzept der kommerziellen Gesundheitsdeterminanten wurde 2016 von Kickbusch und Kollegen formuliert. Gemeint sind damit jene Strategien und Vorgehensweisen der privaten Industrie, welche den Konsum gesundheitsschädlicher Produkte vorantreiben [30].
Die wirtschaftlich mächtige, politisch einflussreiche, globale Zuckerindustrie ist ein Paradebeispiel für kommerziellen Einfluss mit negativen Folgen für die Gesundheit der Bevölkerung. Zucker ist billig und die Gewinnspannen sind hoch. Die Strategien der Industrie ähneln denen der Hersteller anderer gesundheitsschädlicher Produkte, wie Tabak und Alkohol, und sind in den letzten Jahren mehrfach beschrieben worden $[31,32]$. Werbung und aggressives Marketing machen einen bedeutenden Teil der Vermarktungsstrategien aus [33]. Fast jeder kennt die eine oder andere Darstellung der Ernährungspyramide, wobei fettreiche und süße, als ungesund geltende Nahrungsmittel in der Spitze der Pyramide dargestellt werden, um $\mathrm{zu}$ vermitteln, dass sie nur einen sehr geringen Anteil der Nahrung ausmachen sollten. Würde man jedoch eine Pyramide aller Nahrungsmittel zeichnen, wie sie anteilig in der Werbung vorkommen, würden dieselben ungesunden Produkte fast die gesamte Pyramide einnehmen [34]. Besonders problematisch ist die gezielte Vermarktung stark zuckerhaltiger Produkte, die sich direkt an Kinder richtet. Laut einer Untersuchung des Max Rubner-Instituts weisen z. B. speziell als Produkte für Kinder vermarktete Frühstückszerealien und Milchprodukte häufig einen besonders hohen Zuckergehalt auf [18].
Weniger offenkundig sind Strategien, die auf gesellschaftlichen und politischen Einfluss und die öffentliche Wahrnehmung der Marke abzielen („soft power"). Wirtschaftliche Interessen werden mittels Lobbyings der Politik oder des Platzierens von Interessenvertretern in Entscheidungsgremien verfolgt [35]. Vor der Veröffentlichung des WHO-Berichts zur gesunden Ernährung im Jahr 2003, in dem bereits damals ein Zuckerkonsum von maximal 10 Energieprozent empfohlen wurde, forderte die Zuckerindustrie zum Beispiel vom Kongress der Vereinigten Staaten, der WHO die finanziellen Mittel zu streichen [36]. $\mathrm{Zu}$ den Industrietaktiken gehören auch Versuche, wissenschaftliche Forschung zu beeinflussen, die Effektivität von staatlichen Regulierungen in Zweifel zu ziehen und stattdessen auf Selbstregulierung zu pochen, die Imagepflege, z. B. durch das Sponsern von Sport- und Kulturveranstaltungen oder Gesundheitskampagnen, sowie die ständige Betonung der persönlichen Verantwortung des Verbrauchers [37].

Aus diesen Beispielen wird deutlich, dass die Zuckerindustrie bei der Wahrnehmung ihrer Profitinteressen einen erheblichen Einfluss auf das Ernährungsverhalten und damit auf die Gesundheit der Bevölkerung ausübt. Diese Strategien müssen von der Gesundheitspolitik erkannt werden, um ihnen effektiv entgegenwirken zu können.

\section{Implikationen und Strategien für die Gesundheitspolitik}

Die WHO-Richtlinie zum Zuckerkonsum wurde angesichts des wissenschaftlichen Konsenses über die kausale Rolle freier Zucker bei der Entstehung von Übergewicht und Zahnkaries entwickelt. Einzelne Staaten stehen nun vor der Herausforderung, Bedingungen zu schaffen, unter denen diese Empfehlungen erreicht werden können. Es besteht ein dringender Handlungsbedarf.

Ein Großteil der Gesundheitspolitik konzentriert sich nach wie vor auf den Versuch, den einzelnen Verbraucher oder Patienten durch Kenntnisvermittlung zu gesünderem Verhalten zu bewegen. Leider haben bisherige Evaluierungen ge- 


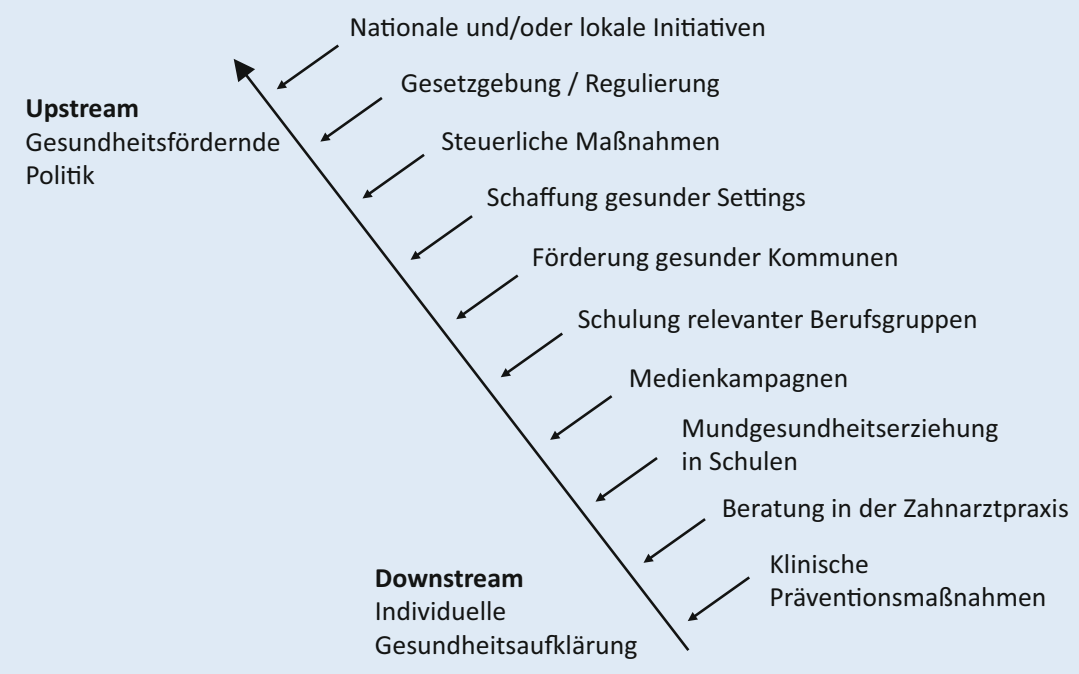

Abb. 3 \ Upstream-Downstream-Ansätze zur Prävention von Zahn- und Munderkrankungen. (Abbildung übersetzt aus Watt [40])

zeigt, dass derartige Interventionen bestenfalls kurzfristig erfolgreich sind [38] und soziale Ungleichheiten sogar verstärken können. Zum Beispiel zeigte die Evaluierung einer informationsbasierten Mundgesundheitskampagne für Schulkinder in Schottland, dass Verbesserungen bei Mundhygiene und Zahnfleischzustand nur bei Kindern in den weniger sozial benachteiligten Schulen erreicht wurden [39]. Das Problem einer alleinigen Fokussierung auf individuelle Verhaltensänderung besteht auch darin, dass die Schuld für eine schlechte Gesundheit auf den Einzelnen übertragen wird (,victim blaming"; [40]). Für politische Entscheidungsträger mag das attraktiv sein, da eine derartige Sichtweise die Politik aus der Verantwortung entlässt. Die oben diskutierten sozialen und kommerziellen Einflüsse werden dabei aber nicht berücksichtigt. Das US-amerikanische Institute of Medicine hat es treffend formuliert: „Man kann nicht erwarten, dass Menschen ihr Verhalten einfach ändern, wenn sich so viele Kräfte im sozialen, kulturellen und materiellen Umfeld gegen solche Veränderungen verschworen haben" [41].

Das bedeutet nicht, dass Gesundheitsaufklärung unwichtig ist. Aber leider hat in Deutschland die Zahl der Menschen, die sich im Umgang mit Gesundheitsund Präventionsempfehlungen überfordert fühlen, im Zeitverlauf zugenommen:
Laut einer aktuellen Studie hatten 2 Drittel der Befragten eine geringe Gesundheitskompetenz, welche außerdem sozial ungleich verteilt war [42]. Hieraus wird klar, dass die Bevölkerung Unterstützung braucht und gesundes Verhalten leichter gemacht werden muss.

\section{Prinzipien und Konzepte der Gesundheitsförderung}

\section{Gemeinsamer Risikofaktoren- ansatz}

Ein anerkanntes Prinzip der Mundgesundheitsförderung ist der gemeinsame Risikofaktorenansatz (Common Risk Factor Approach, CRFA; [43]). Der CRFA ist eine ganzheitliche Perspektive in Anerkennung der Tatsache, dass die wichtigsten Risikofaktoren sowohl Mund- als auch Allgemeinerkrankungen gemein sind. Neben Tabak- und Alkoholkonsum gehören dazu eine ungesunde, zuckerreiche Ernährung sowie die bereits beschriebenen sozialen und kommerziellen Gesundheitsdeterminanten. Die Implikation eines solchen Ansatzes ist die Kräftebündelung durch interdisziplinäre Zusammenarbeit, das heißt die Berücksichtigung von sowohl Mundals auch Allgemeingesundheit in Empfehlungen und Präventionsprogrammen [25].

\section{Hochrisikostrategie und Bevölkerungsstrategie}

Geoffrey Rose hat in seinen Ausführungen zu Hochrisiko- und Bevölkerungsstrategien [44] gezeigt, dass bei häufigen chronischen Erkrankungen die Mehrzahl neuer Fälle (Inzidenz) bei denjenigen auftritt, die nicht zur Hochrisikogruppe gehören, weil "Hochrisikogruppe“ das extreme Ende der Verteilungskurve definiert. Hochrisikostrategien versuchen, diejenigen mit der größten Anfälligkeit für die jeweilige Krankheit durch einen Screeningprozess zu identifizieren, um ihnen individuelle Maßnahmen anzubieten, während Bevölkerungsstrategien auf die Ursachen der Krankheit abzielen. Eine erfolgreiche Hochrisikostrategie schneidet demnach den Hochrisikobereich der Verteilungskurve ab, eine erfolgreiche Bevölkerungsstrategie hingegen verschiebt die gesamte Kurve nach links. Bei der Hochrisikostrategie erhält der Einzelne eine Intervention aufgrund seines individuellen Krankheitsrisikos, allerdings setzt dies ein effektives Screening voraus, ist mit erheblichen Kosten verbunden und beseitigt oft nicht die Krankheitsursachen. Bevölkerungsstrategien können weit mehr neue Fälle verhindern als Hochrisikostrategien und daher hocheffektiv sein (Beispiele sind Maßnahmen zur Tabakkontrolle, Impfkampagnen oder die Sicherheitsgurtpflicht). Ein Mittelweg ist die Teilpopulationsstrategie, bei der sich Maßnahmen auf Bevölkerungsgruppen mit erhöhtem Krankheitsrisiko konzentrieren, ohne dass Einzelne ein Screening durchlaufen müssen. Alle 3 Strategien sollten sich auf sinnvolle Weise ergänzen [44]. Da sie mit weitaus größeren Gesundheitsgewinnen einhergehen, sollten Bevölkerungsstrategien angewendet werden, wann immer dies möglich und akzeptabel ist [45].

\section{Upstream- und Downstream- Ansätze der Prävention}

Die Upstream-Downstream-Metapher beschreibt die Idee, dass es effektiver ist, Krankheiten so weit wie möglich „upstream“, also ursächlich an ihrer Quelle, zu bekämpfen. Upstream-Ansätze basieren hauptsächlich auf Bevölkerungsstrategien. Upstream- und 
Infobox 1 Position der

Bundeszahnärztekammer zum

Thema gesunde Ernährung, wortwörtlich zitiert aus [53]

1. Der Verbraucher hat ein Recht auf eine verständliche Lebensmittelkennzeichnung, insbesondere im Hinblick auf die Menge zuckerhaltiger Nahrungsbestandteile und ungünstiger Fettsäuren.

2. Insbesondere Lebensmittel für Kleinkinder sollten deutlich zuckerreduziert, mit einer klaren Lebensmittelkennzeichnung (speziell auf Zucker) versehen sein und deutlichen Beschränkungen für die Lebensmittelwerbung unterliegen.

3. Die Einführung von Sonderabgaben für stark zucker- und/oder säurehaltige sog. Softdrinks ist eine sinnvolle Maßnahme, wie das Beispiel anderer Länder zeigt.

4. Es braucht verbindliche Standards für eine ausgewogene, gesunde Schul- und Kitaverpflegung.

5. Die Verhältnis- und Verhaltensprävention im Bereich der Ernährung sollte durch Maßnahmen der Präventionsgesetzgebung unterstützt werden.

6. Die Gruppenprophylaxe ( $\$ 21$ SGB V) sollte auch zur Vermittlung einheitlicher Standards von Ernährungsempfehlungen genutzt werden.

Downstream-Ansätze wurden im Kontext der Mundgesundheit vorgestellt [40]. Das Ziel ist eine sinnvolle Kombination von Upstream-, Midstream- und Downstream-Interventionen. - Abb. 3 zeigt Beispiele für die verschiedenen Ansätze, wobei Midstream-Strategien sich hauptsächlich auf lokal umsetzbare Maßnahmen in Zusammenarbeit mit u. a. öffentlichen Einrichtungen, Arbeitgebern und Einzelhandel beziehen [40].

\section{Upstream-, Midstream- und Downstream-Ansätze zur Reduzierung des Zuckerkonsums}

\section{Upstream}

Jahrzehntelange Erfahrungen mit der Tabakindustrie haben gelehrt, dass eine Kombination verschiedener Präventionsmaßnahmen notwendig ist und dass die wirksamsten Strategien auf ganzen Bevölkerungsansätzen und gesetzlichen Regulierungen beruhen [46]. Erfahrungen andernorts haben gezeigt, dass mit freiwilligen Ansätzen, wie sie vom
Bundesministerium für Ernährung und Landwirtschaft (BMEL) bei der Zuckerreduktion favorisiert werden [47], ein substanzieller Fortschritt unwahrscheinlich ist, da die Ziele und Interessen der Industrie den Gesundheitsinteressen der Bevölkerung diametral entgegenstehen. So wurde zum Beispiel Großbritanniens „Responsibility Deal“ von Public-HealthExperten evaluiert und als gescheitert angesehen [48].

Zuckersteuer. Die Besteuerung zuckerhaltiger Getränke in Abhängigkeit von der enthaltenen Zuckermenge ist eine der wirksamsten Maßnahmen zur Reduzierung des Zuckerkonsums und wurde weltweit bereits in mehr als 40 Ländern eingeführt [49]. Die Effektivität von Preiserhöhungen ab $20 \%$ wurde in verschiedenen Evaluations- und Simulationsstudien gezeigt $[49,50]$. So reagierte z. B. im Vereinigten Königreich die Softdrinkindustrie auf die Ankündigung der 2018 eingeführten Zuckersteuer mit der Reduzierung des Zuckeranteils in ihren Getränken um durchschnittlich $38 \%$, der Marktanteil von Getränken mit mehr als $5 \mathrm{~g}$ Zucker pro $100 \mathrm{ml}$ sank um etwa $34 \%$ [51].

In Deutschland wird die Einführung einer „Zucker- oder Soft-Drink-Steuer“ von Ärzten, Zahnärzten und Wissenschaftlern klar befürwortet (siehe - Infobox 1; [52, 53]). Modellrechnungen zeigen, dass die Einführung einer Zuckersteuer in Deutschland zu moderaten Rückgängen sowohl von Karies als auch Übergewicht führen würde. Eine solche Steuer wäre regressiv, d.h., Verbraucher mit geringerem Einkommen wären stärker von den Preissteigerungen betroffen, da sie die besteuerten Produkte im Durchschnitt häufiger konsumieren. Auf der anderen Seite ginge sie aber für genau diese Konsumenten auch mit den größten Gesundheitseffekten einher und könnte somit zur Verringerung sozialer Ungleichheiten beitragen [54, 55]. Die Regressivität der Steuer sollte durch Subventionierung gesünderer Lebensmittel ausgeglichen werden.

Werbeverbote. Die Marketingstrategien der Zuckerindustrie bedürfen wesentlich strikterer Kontrolle als momentan der Fall. Die WHO empfiehlt z.B. seit 2017 ein komplettes Werbeverbot für ungesunde Produkte, die an Kleinkinder gerichtet sind [56]. Aktuell gibt es in Deutschland leider keine verbindlichen Einschränkungen für die Vermarktung ungesunder Lebensmittel an Kinder [57]. Der diesbezügliche Ansatz des BMEL, lediglich Zuckerzusätze in Tees für Babys und Kleinkinder zu verbieten [58], greift viel zu kurz. Eine weitreichendere Regulierung des Marketings von zuckerreichen Lebensmitteln an Kinder, wie von WHO [59] und Robert KochInstitut [60] empfohlen, ist dringend geboten.

Nährwertkennzeichnung. Eine weitere wichtige Upstream-Strategie ist eine obligatorische und leicht verständliche Nährwertkennzeichnung, um Verbrauchern beim Treffen gesünderer Kaufentscheidungen zu helfen. In Deutschland trat im November 2020 die Verordnung zur Einführung des Nutri-Scores (System zur Nährwertkennzeichnung von Lebensmitteln) in Kraft. Dies ist begrüßenswert, ob allerdings der Nutri-Score den gewünschten Effekt haben wird, bleibt fraglich. Denn die Kennzeichnung erfolgt auf freiwilliger Basis, da das geltende EU-Recht eine verpflichtende nationale Anwendung nicht ermöglicht. Innerhalb der EU-Ratspräsidentschaft 2020 setzte sich Deutschland deshalb für eine EU-weit einheitlich erweiterte Nährwertkennzeichnung ein. Derartige Bemühungen sollten von zahnärztlichen Berufsvertretern unterstützt werden.

Präventionsgesetz. Im Jahr 2015 wurde in Deutschland das Präventionsgesetz verabschiedet, welches gesundheitsförderliche Lebenswelten und Verhaltensweisen unterstützen sowie soziale Ungleichheiten von Gesundheitschancen verringern soll. Allerdings findet die Bedeutung freier Zucker für die Mundgesundheit im Gesetz keine Erwähnung. Hier bestehen Chancen für eine Zusammenarbeit im Sinne des gemeinsamen Risikofaktorenansatzes. Bei der geplanten Novellierung des Gesetzes sollten die Mundgesundheitsförderung unbedingt einbezogen und bereits vorhandene Strukturen genutzt werden, wie z.B. die 
zahnmedizinische Gruppenprophylaxe nach $₫ 21$ SGB V (Sozialgesetzbuch, Fünftes Buch).

\section{Midstream}

$\mathrm{Zu}$ den Midstream-Strategien gehört die Schaffung gesundheitsförderlicher Lebenswelten, z.B. in Kitas, Schulen, Betrieben und Krankenhäusern. Gesundheitsfördernde Settings können auch jene Zielgruppen erreichen, die von individuellen verhaltensbezogenen Präventionsangeboten wenig profitieren. Die Deutsche Gesellschaft für Ernährung (DGE) hat für verschiedene Lebenswelten Qualitätsstandards entwickelt, die als Mindeststandards verbindlich gemacht werden sollten [61].

Kitas und Schulen bilden ein ideales Umfeld für die Umsetzung einer (mund-)gesunden Ernährung. Sie können außerdem gesundheitliche Chancengleichheit fördern, vor allem für Kinder aus Familien, in welchen Ernährungsrituale und entsprechende Strukturen fehlen. Eine gesunde, zuckerarme Ernährung zur Prävention von Karies und Übergewicht sollte durch verbindliche, auf Empfehlungen der DGE basierende Richtlinien für Kita- und Schulessen gewährleistet werden. Die Zusammenarbeit mit den pädagogischen Fachkräften, in deren Aufgabenbereich die Gesundheitsbildung in Kita und Schule fällt, ist dabei wesentlich. Ein Beispiel für ein erfolgreiches Kitapräventionsprogramm ist das inzwischen von verschiedenen Landesarbeitsgemeinschaften für Jugendzahnpflege übernommene, ursprünglich Brandenburger GruppenprophylaxeKonzept „Kita mit Biss“ - ein Aufklärungs- und Ernährungsprogramm, das einen mundgesundheitsförderlichen Kitaalltag etablieren soll [62].

Auch im Betrieb können gesundheitliche Rahmenbedingungen für beschäftigte Erwachsene gezielt beeinflusst werden. Auch hier geht es darum, die Verhältnisse am Arbeitsplatz gesundheitsförderlich zu gestalten. Konkret auf die Ernährung bezogen, sollte das Essen am Arbeitsplatz und in der Kantine abwechslungsreich, vollwertig sowie zucker-, fett- und salzarm zubereitet werden. Auf Fertigprodukte sollte verzichtet werden, um versteckten Zuckerverzehr zu vermeiden.
Weitere Möglichkeiten der Integration von Botschaften zur Mundgesundheitsprävention bietet der Nationale Aktionsplan „IN FORM - Deutschlands Initiative für gesunde Ernährung und mehr Bewegung “ [63], der die Prävention von Fehlernährung, Bewegungsmangel, Übergewicht und damit zusammenhängenden Krankheiten zum Ziel hat. Zu den Maßnahmen gehört z. B. die Einrichtung von „Vernetzungsstellen Kita- und Schulverpflegung“ in den Bundesländern zur Förderung von Ernährungswissen in Schulen, welche von der Einbeziehung mundgesundheitlicher Expertise profitieren könnten.

Weitere Midstream-Ansätze sind die Entfernung von Süßwaren aus dem Kassenbereich von Supermärkten, Tankstellen und anderen Geschäften (sogenannte Quengelware) sowie die Regulierung von Preispromotionen für stark zuckerhaltige Produkte [64, 65].

\section{Downstream}

Zahnärztinnen und Zahnärzte sind eine von der Bevölkerung regelmäßig frequentierte und häufig kontrollorientiert in Anspruch genommene Arztgruppe. Deshalb sind die zahnärztlichen Praxen besonders für gesundheitliche Aufklärung und für eine Informationsvermittlung zur gesundheitsförderlichen Verhaltensänderung geeignet [66]. Die Ernährungsberatung durch Zahnärzte und Zahnärztinnen sollte verstärkt werden. Eine solche Beratung muss dabei dem aktuellen Stand der Ernährungsforschung entsprechen und auf evidenzbasierten Methoden beruhen. Um den (versteckten) Zuckerverzehr bei Säuglingen und Kindern zu minimieren, ist außerdem die interdisziplinäre Zusammenarbeit mit Pädiatern, Gynäkologen und Hebammen (Schwangerenberatung) wichtig.

\section{Fazit}

Zucker spielt eine ursächliche Rolle bei der Entwicklung von Karies und Übergewicht und ist daher eine wichtige kommerzielle Gesundheitsdeterminante sowohl für die Mund- als auch die Allgemeingesundheit. Strategien zur Reduzierung des Zuckerkonsums sollten daher disziplinübergreifend und in enger Zusammenarbeit mit anderen Gesundheitsberufen und Public-Health-Akteuren verfolgt werden, um Fachlichkeit und gesundheitspolitischen Einfluss zu bündeln. Prävention ist dann am effektivsten, wenn sie der Rolle sozialer, materieller und kommerzieller Einflüsse Rechnung trägt. Dazu bedarf es einer umfassenden Gesundheitspolitik, welche durch eine sinnvolle Kombination von Upstream-, Midstream- und Downstream-Strategien Lebenswelten schafft, die gesundes Verhalten fördern und erleichtern. Ganz konkret können die verbindliche Einführung einer Ernährungsampel, die Erhebung einer Zuckersteuer auf zuckerhaltige Getränke, Werbeverbote sowie verbesserte Ernährungsprogramme in den Settings Kita und Schule einen entscheidenden Beitrag zur Zuckerreduktion und damit zur Verbesserung der Mund- und Allgemeingesundheit leisten. Die zahnmedizinische Gemeinschaft aus Wissenschaftlern, Praktikern und berufspolitisch Engagierten ist in einer idealen Position, um dabei ihre Expertise einzubringen. Wir möchten eine entsprechende Vernetzung anregen und unterstreichen die Notwendigkeit der Evaluierung existierender Präventionsprogramme sowie weiterer Studien insbesondere zur Effektivität von Midstream- und Downstream-Ansätzen.

\section{Korrespondenzadresse}

\section{Dr. Anja Heilmann}

Department of Epidemiology and Public

Health, University College London

WC1E 6BT London, Großbritannien

anja.heilmann@ucl.ac.uk

Funding. Open access funding provided by University College London (UCL).

\section{Einhaltung ethischer Richtlinien}

Interessenkonflikt. A. Heilmann und S. Ziller geben an, dass kein Interessenkonflikt besteht.

Für diesen Beitrag wurden von den Autoren keine Studien an Menschen oder Tieren durchgeführt. Für die aufgeführten Studien gelten die jeweils dort angegebenen ethischen Richtlinien.

Open Access. Dieser Artikel wird unter der Creative Commons Namensnennung 4.0 International Lizenz veröffentlicht, welche die Nutzung, Vervielfältigung, 
Bearbeitung, Verbreitung und Wiedergabe in jeglichem Medium und Format erlaubt, sofern Sie den/die ursprünglichen Autor(en) und die Quelle ordnungsgemäß nennen, einen Link zur Creative Commons Lizenz beifügen und angeben, ob Änderungen vorgenommen wurden.

Die in diesem Artikel enthaltenen Bilder und sonstiges Drittmaterial unterliegen ebenfalls der genannten Creative Commons Lizenz, sofern sich aus der Abbildungslegende nichts anderes ergibt. Sofern das betreffende Material nicht unter der genannten Creative Commons Lizenz steht und die betreffende Handlung nicht nach gesetzlichen Vorschriften erlaubt ist, ist für die oben aufgeführten Weiterverwendungen des Materials die Einwilligung des jeweiligen Rechteinhabers einzuholen.

Weitere Details zur Lizenz entnehmen Sie bitte der Lizenzinformation auf http://creativecommons.org/ licenses/by/4.0/deed.de.

\section{Literatur}

1. WHO (2021) EB148.R1: Oral Health. World Health Organization. https://apps.who.int/gb/ebwha/ pdf_files/EB148/B148_R1-en.pdf. Zugegriffen: 16. März 2021

2. Kassebaum NJ, Smith AGC, Bernabé E, Fleming TD, Reynolds AE, Vos T, Murray CJL, Marcenes W (2017) Global, regional, and national prevalence, incidence, and disability-adjusted life years for oral conditions for 195 countries, 1990-2015: a systematic analysis for the global burden of diseases, injuries, and risk factors. J Dent Res 96:380-387. https://doi.org/10.1177/0022034517693566

3. Schwendicke F, Dörfer CE, Schlattmann P, Page LF, Thomson WM, Paris S (2015) Socioeconomic inequality and caries: a systematic review and meta-analysis. J Dent Res 94:10-18. https://doi. org/10.1177/0022034514557546

4. Jordan RA, Micheelis W (2016) Fünfte Deutsche Mundgesundheitsstudie (DMSV). Köln: Deutscher Zahnärzte Verlag. https://www.idz.institute/ fileadmin/Content/Publikationen-PDF/Bd_35Fuenfte_Deutsche_Mundgesundheitsstudie_ DMS_V.pdf.Zugegriffen: 16. März 2021

5. Sheiham A (2005) Oral health, general health and quality of life. Bull World Health Organ 83:644

6. Sheiham A, James WPT (2015) Diet and dental caries: the pivotal role of free sugars reemphasized. J Dent Res. https://doi.org/10.1177/ 0022034515590377

7. Moynihan PJ, Kelly SAM (2014) Effect on caries of restricting sugars intake: systematic review to inform WHO guidelines. J Dent Res 93:8-18. https://doi.org/10.1177/0022034513508954

8. Heilmann A, Machuca Vargas C, Watt RG (2021) Sugar consumption and oral health. In: Peres MA, Antunes JLF, Watt RG (Hrsg) Oral epidemiology: a textbook on oral health conditions, research topics and methods. Springer, Cham, S 307-317

9. Chapple ILC, Bouchard P, Cagetti MG, Campus G, Carra M-C, Cocco F, Nibali L, Hujoel P, Laine ML, Lingström $P$ et al (2017) Interaction of lifestyle, behaviour or systemic diseases with dental caries and periodontal diseases: consensus report of group 2 of the joint EFP/ORCA workshop on the boundaries between caries and periodontal diseases. J Clin Periodontol 44:S39-S51. https:// doi.org/10.1111/jcpe.12685

10. Meier T, Deumelandt P, Christen O, Stangl Gl, Riedel K, Langer M (2017) Global burden of sugar- related dental diseases in 168 countries and corresponding health care costs. J Dent Res 96:845-854. https://doi.org/10.1177/0022034517708315

11. Te Morenga L, Mallard S, Mann J (2013) Dietary sugars and body weight: systematic review and meta-analyses of randomised controlled trials and cohort studies. BMJ. https://doi.org/10.1136/bmj. e7492

12. Lean MEJ, Te Morenga $L$ (2016) Sugar and type 2 diabetes. Br Med Bull 120:43-53. https://doi.org/ 10.1093/bmb/ldw037

13. Te Morenga LA, Howatson AJ, Jones RM, Mann J (2014) Dietary sugars and cardiometabolic risk: systematic review and meta-analyses of randomized controlled trials of the effects on blood pressure and lipids. Am J Clin Nutr 100:65-79. https://doi.org/10.3945/ajcn.113.081521

14. WHO (2015) Guideline: sugars intake for adults and children. World Health Organization, Geneva

15. Breda J, Jewell J, Keller A (2019) The importance of the world health organization sugar guidelines for dental health and obesity prevention. Caries Res 53:149-152. https://doi.org/10.1159/000491556

16. Ernst JB, Arens-Azevído U, Bitzer B, Bosy-Westphal A, Zwaan M, Egert S, Fritsche A, Gerlach S, Hauner H, Heseker H et al (2019) Quantitative Empfehlung zur Zuckerzufuhr in Deutschland: Kurzfassung des Konsensuspapiers der Deutschen Adipositas-Gesellschaft e.V. (DAG), der Deutschen Diabetes Gesellschaft e.V. (DDG) und der Deutschen Gesellschaft für Ernährung e. V. (DGE). https://www.ernaehrungs-umschau de/fileadmin/Ernaehrungs-Umschau/pdfs/pdf_ 2019/02_19/EU02_2019_M78-M86.pdf. Zugegriffen: 16. März 2021

17. Heuer T (2018) Zuckerkonsum in Deutschland. Aktuel Ernahrungsmed 43:S8-S11. https://doi. org/10.1055/a-0659-8828

18. Rubner-Institut M (2016) Reformulierung von verarbeiteten Lebensmitteln - Bewertungen und Empfehlungen zur Reduktion des Zuckergehalts. Bundesforschungsinstitut für Ernährung und Lebensmittel. https://www.openagrar. de/servlets/MCRFileNodeServlet/openagrar_ derivate_00017257/S2029_Reformulierung_ Zucker.pdf.Zugegriffen: 16. März 2021

19. Perrar I, Schmitting S, Corte DKW, Buyken AE, Alexy U (2020) Age and time trends in sugar intake among children and adolescents: results from the DONALD study. Eur J Nutr 59:1043-1054. https:// doi.org/10.1007/s00394-019-01965-y

20. Mensink G, Schienkiewitz A, Rabenberg $M$ Borrmann A, Richter A, Haftenberger M (2018) Konsum zuckerhaltiger Erfrischungsgetränke bei Kindern und Jugendlichen in Deutschland Querschnittergebnisse aus KiGGS Welle 2 und Trends. Robert Koch-Institut, Epidemiologie und Gesundheitsberichterstattung, Berlin

21. Popkin BM, Adair LS, Ng SW (2012) Global nutrition transition and the pandemic of obesity in developing countries. Nutr Rev 70:3-21. https:// doi.org/10.1111/j.1753-4887.2011.00456.x

22. Yevlahova D, Satur J (2009) Models for individual oral health promotion and their effectiveness: a systematic review. Aust Dent J 54:190-197. https://doi.org/10.1111/j.1834-7819.2009.01118. $x$

23. de Silva AM, Hegde $S$, Akudo Nwagbara B, Calache H, Gussy MG, Nasser M, Morrice HR, Riggs E, Leong PM, Meyenn LK et al (2016) Community-based population-level interventions for promoting child oral health. Cochrane Database Syst Rev. https://doi.org/10.1002/ 14651858.CD009837.pub2
24. Sheiham A (2000) Improving oral health for all: focusing on determinants and conditions. Health Educ J 59:351-363. https://doi.org/10. 1177/001789690005900408

25. Heilmann A, Sheiham A, Watt RG, Jordan RA (2016) Common Risk Factor Approach - Ein integrierter bevölkerungsbezogener und evidenzbasierter Ansatz zum Ausgleich sozialer Ungleichheiten in der Mundgesundheit. Gesundheitswesen 78:672-677. https://doi.org/10.1055/s-00351548933

26. Solar O, Irwin A (2010) A Conceptual framework for action on the social determinants of health. Geneva: World Health Organization. http:// www.who.int/social_determinants/corner/en/. Zugegriffen: 16. März 2021

27. WHO (2004) Food and health in Europe: a new basis for action. Geneva: world health organization. p.166. https://www.euro.who.int/ en/publications/abstracts/food-and-health-ineurope-a-new-basis-for-action. Zugegriffen: 16. März 2021

28. Jones NRV, Conklin Al, Suhrcke M, Monsivais $P$ (2014) The growing price gap between more and less healthy foods: analysis of a novel longitudinal UK dataset. Plos One 9:e109343. https://doi.org/ 10.1371/journal.pone.0109343

29. van Strien T (2018) Causes of emotional eating and matched treatment of obesity. Curr Diab Rep 18:35-35. https://doi.org/10.1007/s11892-0181000-x

30. Kickbusch I, Allen L, Franz C (2016) The commercial determinants of health. Lancet Glob Health 4:e895-e896. https://doi.org/10.1016/S2214109X(16)30217-0

31. Barlow P, Serodio P, Ruskin G, McKee M, Stuckler D (2018) Science organisations and Coca-Cola's "war" with the public health community: insights from an internal industry document. J Epidemiol Community Health 72:761-763. https://doi.org/ 10.1136/jech-2017-210375

32. Peres MA, Macpherson LMD, Weyant RJ, Daly B, Venturelli R, Mathur MR, Listl S, Celeste RK, Guarnizo-Herreño CC, Kearns C et al (2019) Oral diseases: a global public health challenge. Lancet 394:249-260. https://doi.org/10.1016/S01406736(19)31146-8

33. Capewell S, Lloyd-Williams F (2018) The role of the food industry in health: lessons from tobacco? $\mathrm{Br}$ Med Bull 125:131-143. https://doi.org/10.1093/ bmb/ldy002

34. Dalmeny EH, Lobstein T (2003) Broadcasting bad health: Why food marketing to children needs to be controlled. A report by the International Association of Consumer Food Organizations for the World Health Organization consultation on a global strategy for diet and health. IAFCO. http://www.foodcomm.org.uk/ pdfs/Broadcasting_bad_health.pdf. Zugegriffen: 16. März 2021

35. McKee M, Stuckler D (2018) Revisiting the corporate and commercial determinants of health. Am J Public Health 108:1167-1170. https://doi. org/10.2105/AJPH.2018.304510

36. Boseley S, McMahon J (2003) Political context of the world health organization: sugar industry threatens to scupper the WHO. Int J Health Serv 33:831-833. https://doi.org/10.2190/u0mwwm82-n5bh-e20c

37. Knai C, Petticrew M, Mays N, Capewell S, Cassidy R, Cummins S, Eastmure E, Fafard $P$, Hawkins B, Jensen JD et al (2018) Systems thinking as a framework for analyzing commercial 


\section{Leitthema}

determinants of health. Milbank 0 96:472-498. https://doi.org/10.1111/1468-0009.12339

38. Watt RG, Marinho VC (2005) Does oral health promotion improve oral hygiene and gingival health? Periodontol 2000 37:35-47. https://doi. org/10.1111/j.1600-0757.2004.03796.X

39. Schou L, Wight C (1994) Does dental health education affect inequalities in dental health? Community Dent Health 11:97-100

40. Watt RG (2007) From victim blaming to upstream action: tackling the social determinants of oral health inequalities. Community Dent Oral Epidemiol 35:1-11. https://doi.org/10.1111/j. 1600-0528.2007.00348.x

41. Institute of Medicine (2000) Promoting health intervention strategies from social and behavioral research. The National Academies Press, Washington, DChttps://doi.org/10.17226/9939

42. Hurrelmann K, Klinger J, Schaeffer D (2020) Gesundheitskompetenz der Bevölkerung in Deutschland: Vergleich der Erhebungen 2014 und 2020. Universität Bielefeld, Interdisziplinäres Zentrum für Gesundheitskompetenzforschung (IZKG), Bielefeld (https://pub.uni-bielefeld.de/ download/2950303/2950404/HLS-GER\%201_ 2020.pdf Zugriffsdatum: 16/03/2021)

43. Watt RG, Sheiham A (2012) Integrating the common risk factor approach into a social determinants framework. Community Dent Oral Epidemiol 40:289-296. https://doi.org/10.1111/j. 1600-0528.2012.00680.x

44. Rose G (2001) Sickindividuals and sick populations. Int J Epidemiol 30:427-432. https://doi.org/10. 1093/ije/30.3.427

45. Mackenbach JP, Lingsma HF, van Ravesteyn NT, Kamphuis CB (2013) The population and high-risk approaches to prevention: quantitative estimates of their contribution to population health in the Netherlands, 1970-2010. Eur J Public Health 23:909-915. https://doi.org/10.1093/eurpub/ cks106

46. Levy DT, Tam J, Kuo C, Fong GT, Chaloupka F (2018) The impact of implementing tobacco control policies: the 2017 tobacco control policy scorecard. J Public Health Manag Pract 24:448-457. https:// doi.org/10.1097/phh.0000000000000780

47. Bundesministerium für Ernährung und Landwirtschaft (BMEL) (2018) Nationale Reduktions- und Innovationsstrategie für Zucker, Fette und Salz in Fertigprodukten. Bonn: Bundesministerium für Ernährung und Landwirtschaft. https://www.bmel. de/SharedDocs/Downloads/DE/Broschueren/ NationaleReduktionsInnovationsstrategieLayout.pdf?_blob=publicationFile\&v=4. Zugegriffen: 16. März 2021

48. Knai $C$, Petticrew M, Durand MA, Eastmure E, James L, Mehrotra A, Scott C, Mays N (2015) Has a public - private partnership resulted in action on healthier diets in England? An analysis of the Public Health Responsibility Deal food pledges. Food Policy 54:1-10. https://doi.org/10.1016/j.foodpol. 2015.04.002

49. World Bank (2020) Taxes on sugar-sweetened beverages: international evidence and experiences. World bank. https://openknowledge.worldbank. org/handle/10986/33969. Zugegriffen: 16. März 2021

50. Jevdjevic M, Trescher AL, Rovers M, Listl S (2019) The caries-related cost and effects of a tax on sugarsweetened beverages. Public Health 169:125-132. https://doi.org/10.1016/j.puhe.2019.02.010

51. Scarborough $P$, Adhikari $V$, Harrington RA Elhussein A, Briggs A, Rayner $M$, Adams J, Cummins S, Penney T, White M (2020) Impact of the announcement and implementation of the UK Soft Drinks Industry Levy on sugar content, price, product size and number of available soft drinks in the UK, 2015-19: A controlled interrupted time series analysis. PLoS Med 17:e1003025.https://doi. org/10.1371/journal.pmed.1003025

52. Berufsverband der Kinder- und Jugendärzte e.V (2020) AOK, BVKJ und DDG fordern gesetzgeberische Maßnahmen zur Zuckerreduktion. https://www.bvkj.de/politik-und-presse/ nachrichten/2-2020-10-27-aok-bvkj-und-ddgfordern-gesetzgeberische-massnahmen-zurzuckerreduktion.Zugegriffen: 16. März 2021

53. Bundeszahnärztekammer (2018) Position: Bedeutung der Ernährung im Rahmen zahnmedizinischer Erkrankungen. Berlin: Bundeszahnärztekammer. https://www.bzaek.de/fileadmin/PDFs/ b/Position_Ernaehrung.pdf.Zugegriffen: 16. März 2021

54. Schwendicke F, Thomson WM, Broadbent JM, Stolpe M (2016) Effects of taxing sugar-sweetened beverages on caries and treatment costs. JDentRes 95:1327-1332.https://doi.org/10.1177/ 0022034516660278

55. Schwendicke F, Stolpe M (2017) Taxing sugarsweetened beverages: impact on overweight and obesity in Germany. BMC Public Health 17:88. https://doi.org/10.1186/s12889-016-3938-4

56. WHO (2017) Guidance on ending the inappropriate promotion of foods for infants and young children: implementation manual. World Health Organization, Geneva (https://www.who.int/ nutrition/publications/infantfeeding/manualending-inappropriate-promotion-food/en/ Zugriffsdatum: 16/03/2021)

57. Robert Koch-Institut (RKI) (2020) AdiMon-Themenblatt: Policy-Maßnahmen (Stand: 1. Oktober 2020) (www.rki.de/adimon)

58. Bundesgesetzblatt Jahrgang 2020. 2020. Vierte Verordnung zur Änderung der Fruchtsaft- und Erfrischungsgetränkeverordnung vom 18. Mai 2020. Bundesgesetzblatt Jahrgang 2020 Teil I, Nr. 24, ausgegeben zu Bonn am 28. Mai 2020: 1075-1076.

59. WHO (2010) Set of recommendations on the marketing of foods and non-alcoholic beverages to children. World Health Organization, Geneva (https://www.who.int/dietphysicalactivity/ publications/recsmarketing/en/ Zugriffsdatum: $16 / 03 / 2021)$

60. Mensink GB, Haftenberger $M$, Barbosa $C L$, Brettschneider AK, Lehmann F, Frank M, Heide $K_{\text {, }}$ Moosburger R, Patelakis E, Perlitz H (2020) EsKiMo II - Die Ernährungsstudie als KiGGSModul. Robert Koch-Institut, Berlin (https://edoc rki.de/bitstream/handle/176904/6887/EsKiMoll Projektbericht_2814HS004.pdf?sequence $=1 \&$ isAllowed=y Zugriffsdatum: 16/03/2021)

61. Deutsche Gesellschaft für Ernährung e. V. (2021) DGE-Qualitätsstandards. https://www.dge.de/gv/ dge-qualitaetsstandards/. Zugegriffen: 16. März 2021

62. HaakP, Rojas G (2013) Kita mit Biss - Frühkindlicher Karies vorbeugen. Zahnärztl Mitt 11:42-43

63. Bundesministerium für Ernährung und Landwirtschaft, Bundesministerium für Gesundheit (2014) IN FORM - Nationaler Aktionsplan zur Prävention von Fehlernährung, Bewegungsmangel, Übergewicht und damit zusammenhängenden Krankheiten. Berlin: BMEL. https://www.in-form. de/fileadmin/Dokumente/Materialien/IN_FORMNationaler_Aktionsplan.pdf.Zugegriffen:16.März 2021
64. Ejlerskov KT, Sharp SJ, Stead M, Adamson AJ, White M, Adams J (2018) Supermarket policies on less-healthy food at checkouts: Natural experimental evaluation using interrupted time series analyses of purchases. PLoS Med 15:e1002712. https://doi.org/10.1371/journal.pmed.1002712

65. Watt TL, Beckert W, Smith RD, Cornelsen L (2020) Reducing consumption of unhealthy foods and beverages through banning price promotions: what is the evidence and will it work? Public Health Nutr 23:2228-2233. https://doi.org/10. $1017 / \mathrm{s} 1368980019004956$

66. Ziller S, Oesterreich D (2007) „Dental Public Health“ in Deutschland - eine Bestandsaufnahme. Präv Gesundheitsf 2:31-38. https://doi.org/10.1007/ s11553-006-0043-z 